\title{
Should RECOVERY have used Response Adaptive Randomization? Evidence from a simulation study
}

Tamir Sirkis ( $\square$ tamir@live.co.uk)

University of Exeter Medical School https://orcid.org/0000-0002-2631-6105

Jack Bowden

University of Exeter Medical School

Benjamin Jones

PenARC: NIHR Applied Research Collaboration South West Peninsula

\section{Research Article}

Keywords: COVID-19, RECOVERY, response-adaptive randomization, REMAP-CAP, coronavirus, adaptive trial, platform trial, simulation

Posted Date: August 16th, 2021

DOI: https://doi.org/10.21203/rs.3.rs-814621/v1

License: (c) (i) This work is licensed under a Creative Commons Attribution 4.0 International License. Read Full License 


\section{Evidence from a simulation study}

Tamir Sirkis ${ }^{1^{*}}$, tamir@live.co.uk; Benjamin Jones, b.g.jones@exeter.ac.uk ${ }^{2}$; Jack Bowden, j.bowden2@exeter.ac.uk ${ }^{1}$

1. University of Exeter College of Medicine and Health, Exeter, U.K.

2. NIHR ARC South West Peninsula (PenARC), College of Medicine and Health, University of Exeter

*corresponding author, tamir@live.co.uk 


\section{Abstract}

\section{Background}

The Randomised Evaluation of COVID-19 Therapy (RECOVERY) trial is aimed at addressing the urgent need to find effective treatments for patients hospitalised with suspected or confirmed COVID-19. The trial has had many successes, including discovering that dexamethasone is effective at reducing COVID-19 mortality, the first treatment to reach this milestone in a randomised controlled trial. Despite this, it continues to use standard or 'fixed' randomization (FR) to allocate patients to treatments. We assessed the impact of implementing response adaptive randomization (RAR) within RECOVERY using an array of performance measures, to learn if it could be beneficial going forward. This design feature has recently been implemented within the REMAP-CAP trial.

\section{Methods}

Trial data was simulated to closely match the data for patients allocated to either standard care or dexamethasone in the RECOVERY trial from March-June 2020, representing two out of five arms tested throughout this period. Two forms of FR and two forms of RAR were tested. Randomization strategies were performed at the whole trial level as well as within three pre-specified patient subgroups defined by patients' respiratory support level.

\section{Results}

RAR strategies led to more patients being given dexamethasone and a lower mortality rate in the trial. Subgroup specific RAR reduced mortality rates even further. RAR did not induce any meaningful bias in treatment effect estimates, but reduced statistical power compared to FR, with subgroup level adaptive randomizations exhibiting the largest power reduction.

\section{Conclusions}


Using RAR within RECOVERY could have resulted in fewer deaths in the trial. However, a larger trial would have been needed to attain the same study power. This could potentially have prolonged the time to full approval of the drug, unless RAR itself led to an increased recruitment rate. Deciding how to balance the needs of patients within a trial and future patients who have yet to fall ill is an important ethical question of our time. RAR deserves to be considered as a design feature in future trials of COVID-19 and other diseases.

Keywords: COVID-19, RECOVERY, response-adaptive randomization, REMAP-CAP, coronavirus, adaptive trial, platform trial, simulation

\section{Background}

Coronavirus disease 2019 (COVID-19) is a condition caused by the severe acute respiratory syndrome coronavirus 2 (1). On March 11 2020, the global incidence and virulence of COVID-19 met the criteria for the World Health Organisation to declare it a pandemic (2). At the time of writing, the disease has caused 200 million cases and 4 million deaths worldwide (3). Furthermore, some patients who contracted COVID-19 report experiencing long covid, a condition consisting of many symptoms such as profound fatigue that can persist long after the initial infection has passed (4). It has been estimated there are around two million people in the UK who have suffered from long covid (5). The effects of the pandemic have been far reaching and extend beyond just those infected. For example, many patients with suspected cancer are not receiving appropriate early management, which experts believe will lead to increased mortality as their condition remains untreated (6). Likewise, the pandemic has been noted to increase and exacerbate mental health problems such as stress and anxiety (7). In terms of the wider societal impact, the pandemic has also led to a sharp increase in extreme poverty, 1.5 billion students to miss out on education, and increasing food insecurity (8).

When COVID-19 first emerged, very little was known about its pathophysiology and, as a result, clinicians were unsure which treatments would reduce COVID-19's associated morbidity and 
mortality. To address this, large scale randomized clinical trials were quickly designed, authorised, and initiated in patients with severe COVID-19 symptoms in a bid to find effective treatments. One of the most high profile examples is the Randomised Evaluation of COVid-19 ThERapY (RECOVERY) trial (9). It commenced on March 19 2020, eight days after the pandemic announcement, with the aim of discovering new treatments that are effective in reducing 28-day mortality in patients hospitalised with confirmed or suspected COVID-19(9). To date, 15 treatments have been trialled, most consisting of repurposed drugs (9). Nine treatments have had results published, and three of these have been declared successful: dexamethasone (10), tocilizumab(11) and REGENERON (12). When analysing the dexamethasone data, researchers found a statistically significant effect in all patients, where fewer patients died on dexamethasone (22.9\%) compared to standard care (25.7\%) (10). but also performed a stratified analysis in three pre-specified patient subgroups who received different levels of respiratory support at the time of randomization: (i) no oxygen, (ii) oxygen only, and (iii) oxygen through invasive mechanical ventilation. The subgroup analysis uncovered important treatment effect heterogeneity: The most severely ill patients in group (iii) received a larger benefit from taking dexamethasone compared to group (ii), while relatively stable patients in group (i) appeared to fare better with standard care (although this was not statistically significant) (10). These results are shown in Table 1. The National Institute for Health and Care Excellence issued guidelines based on this research, stating dexamethasone should be given to hospitalised patients with COVID19 if they require oxygen (13).

\begin{tabular}{|c|c|c|c|c|c|c|}
\hline $\begin{array}{l}\text { Patient } \\
\text { subgroup }\end{array}$ & $\begin{array}{l}\text { Number } \\
\text { of } \\
\text { patients }\end{array}$ & $\begin{array}{l}\text { Proportion } \\
\text { of patients }\end{array}$ & $\begin{array}{l}\text { Number } \\
\text { allocated } \\
\text { standard } \\
\text { care }\end{array}$ & $\begin{array}{l}\text { Number allocated } \\
\text { dexamethasone }\end{array}$ & $\begin{array}{l}\text { Mortality } \\
\text { on } \\
\text { standard } \\
\text { care }\end{array}$ & $\begin{array}{l}\text { Mortality on } \\
\text { dexamethasone }\end{array}$ \\
\hline No oxygen & 1535 & $23.9 \%$ & 1034 & 501 & $14.0 \%$ & $17.8 \%$ \\
\hline
\end{tabular}




\begin{tabular}{|c|c|c|c|c|c|c|}
\hline $\begin{array}{l}\text { Oxygen- } \\
\text { only }\end{array}$ & 3883 & $60.3 \%$ & 2604 & 1279 & $26.2 \%$ & $23.3 \%$ \\
\hline $\begin{array}{l}\text { Invasive } \\
\text { ventilation }\end{array}$ & 1007 & $15.7 \%$ & 683 & 324 & $41.4 \%$ & $29.3 \%$ \\
\hline
\end{tabular}

Table 1: Mortality data for the RECOVERY trial stratified by patient group.

RECOVERY is an excellent example of a modern adaptive platform trial (14). Unlike a traditional trial where all design aspects (including the treatments to be compared and the sample size) must be decided before the trial commences, adaptive platform trials have the freedom to continue indefinitely. Whilst the trial is ongoing, new experimental treatments can be added and tested, old experimental treatments showing little benefit can be dropped and effective experimental treatments can be 'graduated' to become the de-facto standard of care. Many adaptive trial designs exist, but their common aim is to be more flexible and resource efficient. Supporters of adaptive designs assert that they are more ethical (15), although this is not universally accepted (16). One of the most controversial features that can be incorporated into an adaptive trial is response-adaptive randomization (RAR). RECOVERY chose not to incorporate this, preferring instead to use standard 'fixed' randomization (FR) probabilities when allocating patients to experimental or standard care arms during the trial. Under a RAR scheme, allocation starts in the same way as FR i.e.., by beginning with a fixed allocation ratio to randomise an initial batch of patients. RAR then facilitates the adaptation of the allocation ratio, as interim analyses begin to show that there is a genuine difference between outcomes in the different arms, to favour treatment arms that have a higher estimated probability of a favourable outcome.. A recent example of a COVID-19 study that uses RAR is the Randomised, Embedded, Multi-factorial, Adaptive Platform Trial for Community-Acquired Pneumonia (REMAP-CAP) (17). This is a trial that aims to evaluate multiple interventions 
simultaneously for community acquired pneumonia but has a sub-platform, REMAP-COVID, created to assess COVID-19 treatments (18).

The aim of this paper is to investigate, by simulation, the possible benefit of applying RAR instead of FR to assign patients to either dexamethasone or standard of care in the RECOVERY trial. We hypothesised that applying RAR would reduce the number of deaths amongst trial participants by allocating more patients to their optimal treatment. To implement RAR, we use the REMAP-CAP

\section{Methods} algorithm as well as our own bespoke tuning algorithm. We apply each RAR method across the whole patient cohort and separately within patient subgroups (i)-(iii). We apply FR using allocation ratios of 1:1 and 2:1 (with respect to standard care: dexamethasone), with the latter having been used in RECOVERY. To quantify benefit, we focus on the following four metrics:

- The proportion of patients allocated to each treatment;

- The expected number of deaths throughout trial;

- The statistical power to detect a treatment effect in all patients and in patient subgroups;

- The bias and mean squared error the treatment effect estimate.

\section{Simulation set up}

The parameters of the simulation were selected to closely resemble the observed results of the RECOVERY trial. The original trial followed up a total of 6425 patients across the dexamethasone $(\mathrm{N}=$ 2104) and standard of care $(N=4321)$ arms between March 19 and June 8,2020 . At this point the trial was halted and results of its efficacy were published (10). To provide a means to implement RAR, patient outcome data was simulated in 100 blocks (or stages) of 150 patients to give a total of 15000 patients. Although this sample size exceeds that of the RECOVERY trial, it enables ourstudy to evaluate the statistical properties of the design at both smaller and larger sample sizes than the actual trial. To match RECOVERY, $24 \%, 60 \%$ and $16 \%$ of each block were drawn from patient 
subgroups (i)-(iii), respectively. Patient outcomes $(Y)$ representing the primary outcome of mortality at 28 days were generated from a Bernoulli distribution, where for patient $i$ randomized in block $j$ in patient subgroup $k$, on treatment $l$ :

$$
Y_{i, j, k, l} \sim \operatorname{bern}\left(P_{k, l}\right),
$$

where $i=1, \ldots, 150, j=1, \ldots, 100, \mathrm{k}=\mathrm{i}, \mathrm{ii}, \mathrm{iii}, I=0$ (standard of care) and 1 (standard of care + dexamethasone). The values of $P_{k, l}$ (the 28 day mortality rate) match the rates observed in RECOVERY (see Table 1).

\section{Randomization allocation strategies}

Six allocation strategies (two FR and four RAR) were investigated as part of this simulation. FR was investigated using both a 1:1 and a 2:1 standard care: dexamethasone ratio. Given the underlying outcome rates assumed in each trial arm are similar (Table 1), 1:1 allocation, or a 50\% probability of receiving either, drug is near-optimal in terms of statistical power according to Neyman's rule (19) (the exact value being a 51\%/491\% split). For further details see Technical Appendix A. The latter 1:2 strategy was used in the RECOVERY trial. RAR was investigated using two randomization algorithms, our tuning algorithm $(T)$ and the algorithm used in REMAP-CAP (RMC). We use $T_{f}$ and $R M C_{f}$, to denote the RAR allocation procedures applied to trial patients across the full patient cohort. We use $\mathrm{T}_{\mathrm{s}}$ and $\mathrm{RMC}_{\mathrm{s}}$, to denote RAR allocation procedures applied within each patient subgroup. Specifically, the probability of patients in block $j$ and subgroup $\mathrm{k}$ being allocated to the dexamethasone group given treatment and outcome data on all preceding patients in blocks $1, \ldots, j-1$ is denoted by $\alpha_{\mathrm{j}, \mathrm{k}}$, where: 


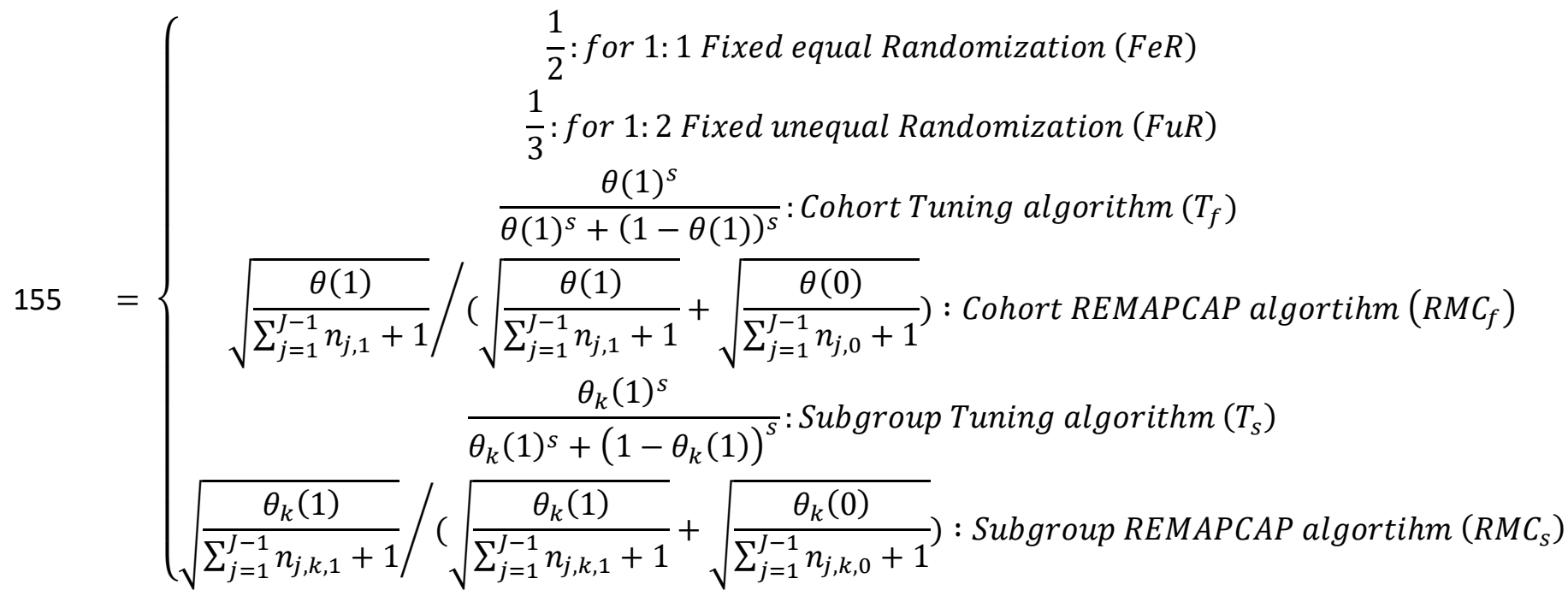

Where $\Theta$ represents the posterior probability that a treatment I is the optimal treatment, either for the full cohort or for subgroup $k$ (further details supplied in Technical Appendix B), $s$ is the proportion of trial stages completed at point of adaption; and $\mathrm{n}$ represents number of patients in stage $\mathrm{k}$ in the cohort/sub-group that have been allocated treatment I.

In addition, allocation probabilities in RAR schemes T and RMC were constrained by a maximum and minimum value according to the following rule:

$$
\alpha_{j, k}=\left\{\begin{array}{c}
0.9 \text { if } \alpha_{j, k}>0.9 \\
\alpha_{j, k} \text { if } 0.1 \leq \alpha_{j, k} \leq 0.9 \\
0.1 \text { if } \alpha_{j, k}<0.1
\end{array}\right.
$$

Both the T and RMC RAR procedures used a "burn-in" period (a period where adaptive

randomization was not applied) for the first two blocks of participants, meaning the first 300 allocated using RAR. Each simulation was performed 1000 times. 
To assess the performance of the methods, the following summary measures were calculated across the 1000 simulated trials:

- The expected or average number of patients allocated to Dexamethasone $E\left[N_{d}\right]$

- The expected or average number of deaths $E\left[N_{Y}\right]$ at point where 6450 patients were allocated to a treatment, a number chosen due to its proximity to the 6425 patients recruited in RECOVERY.

- The expected probability (or statistical power) of rejecting the null hypothesis of a zero treatment effect at the end of each block $j=1, \ldots, 100$ in the trial, $E\left[R e j_{j}\right]$, calculated by $\sum_{t=1}^{T}\left\{\begin{array}{l}R e j_{j}=1 \text { if } p \leq 0.05 \\ R e j_{j}=0 \text { if } p>0.05\end{array} / T\right.$ for trials $\mathrm{t}=1, \ldots, 1000$. This was calculated using a logistic regression model.

- The expected estimation error (or bias) in treatment effect estimation bias $_{k}$, where bias $_{k}=E\left[\frac{\left(\hat{P}_{k, l}-\hat{P}_{k, l}\right)-\left(P_{k, 0}-P_{k, 1}\right)}{\left(P_{k, 0}-P_{k, 1}\right)}\right]$, and where $P_{k, l}$ is the actual mortality rate and $\hat{P}_{k, l}$ is the estimated mortality rate in subgroup $k$ (1=i, 2=ii, 3=iii) on treatment / ( 1 for dexamethasone, 0 for standard of care) from the trial. This will only be done for RAR procedures, as only RAR is associated with treatment effect bias as explained in technical appendix $C$.

\section{Summary}

To summarise, the simulation study investigated six treatment allocation methods:

- 1:1 FR across all patients (FeR)

- 1:2 FR across all patients (FuR)

- T algorithm across all patients $\left(T_{f}\right)$

- $\quad \mathrm{RMC}$ algorithm across all patients $\left(\mathrm{RMC}_{\mathrm{f}}\right)$

- T algorithm within subgroups i-iii separately $\left(T_{s}\right)$

- $\mathrm{RMC}$ algorithm within subgroups i-iii separately $\left(\mathrm{RMC}_{\mathrm{s}}\right)$ 

number of deaths, $E\left[N_{Y}\right]$, is quantified under four theoretical strategies shown in Table 2. and therefore should be compared with $\mathrm{T}_{\mathrm{f}}$ and $\mathrm{RMC}_{\mathrm{f}}$ allocation strategies. In contrast, strategies 3

\begin{tabular}{|c|c|c|}
\hline Strategy & Description & $\begin{array}{l}\text { Expected } \\
\text { mortality (\%) }\end{array}$ \\
\hline 1. Dexamethasone & All patients given dexamethasone & $1,477(22.9 \%)$ \\
\hline 2. Standard Care & All patients given standard care & $1,655(25.7 \%)$ \\
\hline $\begin{array}{l}\text { 3. Sub-group } \\
\text { optimal }\end{array}$ & $\begin{array}{l}\text { No oxygen group given standard care; other } \\
\text { groups given dexamethasone }\end{array}$ & $1,419(22.0 \%)$ \\
\hline $\begin{array}{l}\text { 4. Sub-group least } \\
\text { optimal }\end{array}$ & $\begin{array}{l}\text { No oxygen group given dexamethasone; other } \\
\text { groups given standard care }\end{array}$ & $1,713(26.6 \%)$ \\
\hline
\end{tabular}

Table 2: Theoretical strategies leading to the best and worst mortality rates on patients given standard care and dexamethasone

\section{Results}

\section{Allocation ratios}

Both cohort RAR methods led to more patients receiving dexamethasone compared to either FR approach. $\mathrm{T}_{\mathrm{f}}$ led to slightly more patients receiving dexamethasone than $\mathrm{RMC}_{\mathrm{f}}$. When considering subgroup-specific RAR, each subgroup has its own trend. In subgroup (i), both adaptive methods allocate slightly more patients to standard care compared to FuR, with $T_{s}$ allocating standard care to the most patients. In subgroups (ii) and (iii), the RAR algorithms allocate mainly to dexamethasone, 
with $\mathrm{RMC}_{\mathrm{s}}$ allocating dexamethasone to the most patients. However, the allocation disparity is much larger in the subgroup (iii). These differences are demonstrated in Figure 1.

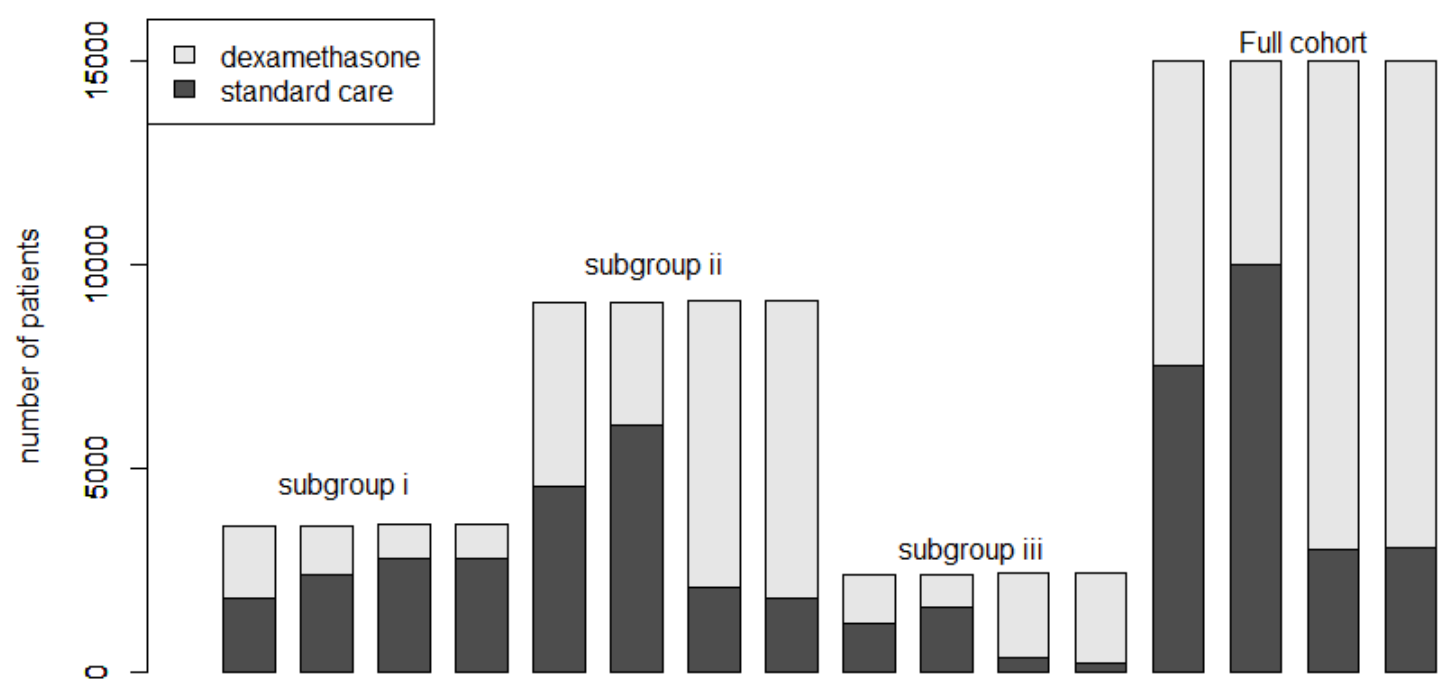

FeR FuR $T_{s} \quad R M C_{s}$ FeR fuR $T_{s} \quad R M C_{s}$ FeR FuR $T_{s} \quad R M C_{s}$ FeR FuR $T_{f} \quad R M C_{f}$

Randomisation algorithm and sub-group

The RAR algorithms also differ in how they "ramp up" allocation to the optimal treatment as the trial progresses. $T_{f}$ increases randomization to the optimal treatment at a steadier rate throughout the trial, whereas $\mathrm{RMC}_{\mathrm{f}}$ increases randomization faster in the early trial stages but also plateaus earlier.

217 This is consistent with the pattern in subgroup-level RAR methods, $T_{s}$ and $R M C_{s}$, for subgroups (i)

218 and (ii). However, in subgroup (iii), the $\mathrm{RMC}_{\mathrm{s}}$ algorithm ramps up allocation to dexamethasone faster across the whole trial. This is demonstrated in Figures 2 and 3. 


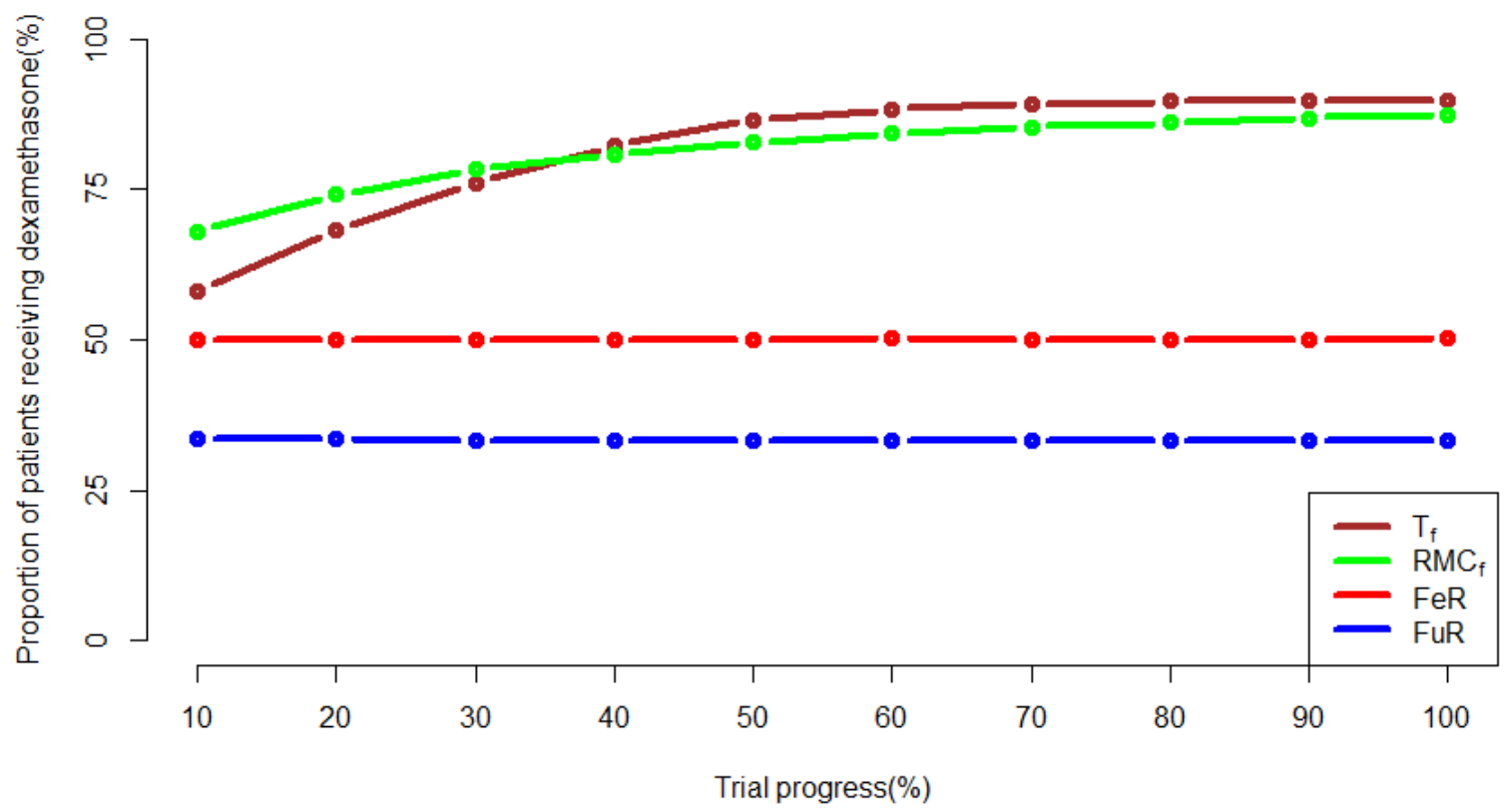

221

222 Figure 2:The proportion of patients receiving dexamethasone across the trial for FR and cohort-level RAR frameworks.

223 Vertical line at 43\% trial progress represents the observed sample size of RECOVERY (6425). 


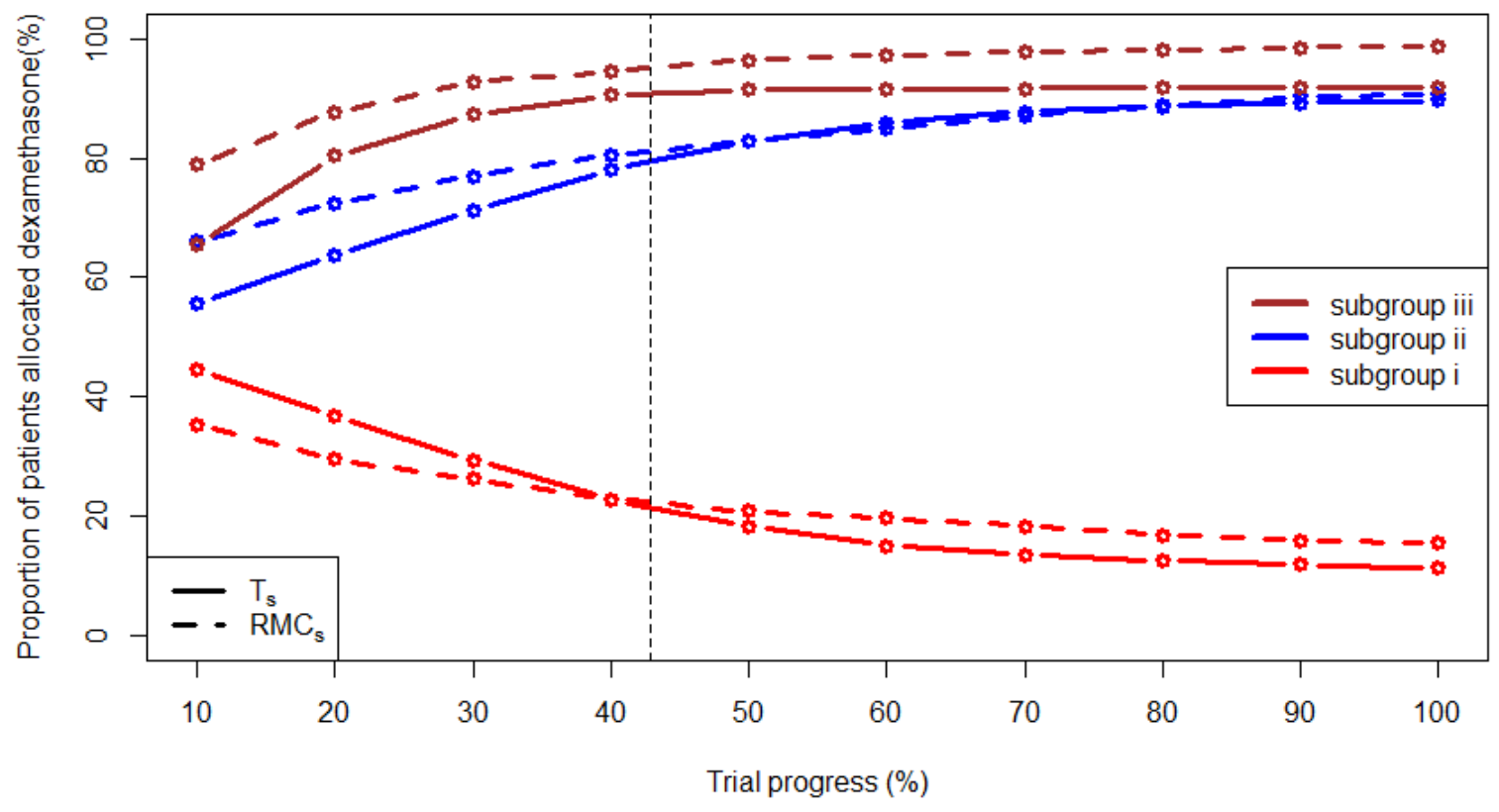

225

Figure 3: the allocation of patients to dexamethasone and no treatment in $T_{s}$ and $R M C_{s}$. Vertical line at $43 \%$ trial progress represents the observed sample size of RECOVERY (6425)..

Mortality rates

FR led to the highest number of expected deaths (with FuR being worst than FeR). This was followed by cohort-level RAR, with the lowest mortality rates observed when using subgroup-specific RAR, with $\mathrm{RMC}_{\mathrm{f}}$ being worse than $\mathrm{T}_{\mathrm{f}}$. Finally, sub-group specific RAR led to the fewest deaths, with $\mathrm{T}_{\mathrm{s}}$ being better than $\mathrm{RMC}_{\text {s. }}$ The expected mortality figures are given in Table 3, expressed as deaths prevented relative to FuR.

\begin{tabular}{|l|l|l|}
\hline Randomization strategy & $\begin{array}{l}\text { Average no. of deaths at } \mathbf{n}= \\
\mathbf{6 4 5 0} \text { (percentage) }\end{array}$ & $\begin{array}{l}\text { Deaths prevented compared } \\
\text { to FuR at } \mathbf{n}=\mathbf{6 4 5 0}\end{array}$ \\
\hline FuR & $1596(24.8 \%)$ & 0 \\
\hline FeR & $1569(24.3 \%)$ & 27 \\
\hline
\end{tabular}




\begin{tabular}{|l|l|l|}
\hline$T_{f}$ & $1534(23.5 \%)$ & 62 \\
\hline RMC $_{f}$ & $1526(23.4 \%)$ & 70 \\
\hline$T_{s}$ & $1520(22.9 \%)$ & 76 \\
\hline RMC $_{s}$ & $1501(23.1 \%)$ & 95 \\
\hline
\end{tabular}

Table 3: Average mortality rates in whole trial for each randomization method, given at point that RECOVERY

trial was finished.

Statistical power

FeR leads to the highest study power on a cohort level, reaching $80 \%$ and $90 \%$ power with fewer patients than any other randomization procedure, as expected by Neyman's rule. FuR, $T_{f}$ and $R M C_{f}$ all performed similarly, with FuR having slightly more power by the end of the simulation and at the point where the simulation has almost the same sample size as RECOVERY. Comparing the cohort RAR algorithms, $T_{f}$ performs slightly better earlier in most points of the trial than $R M C_{f}$. These results are shown in Figure 4.

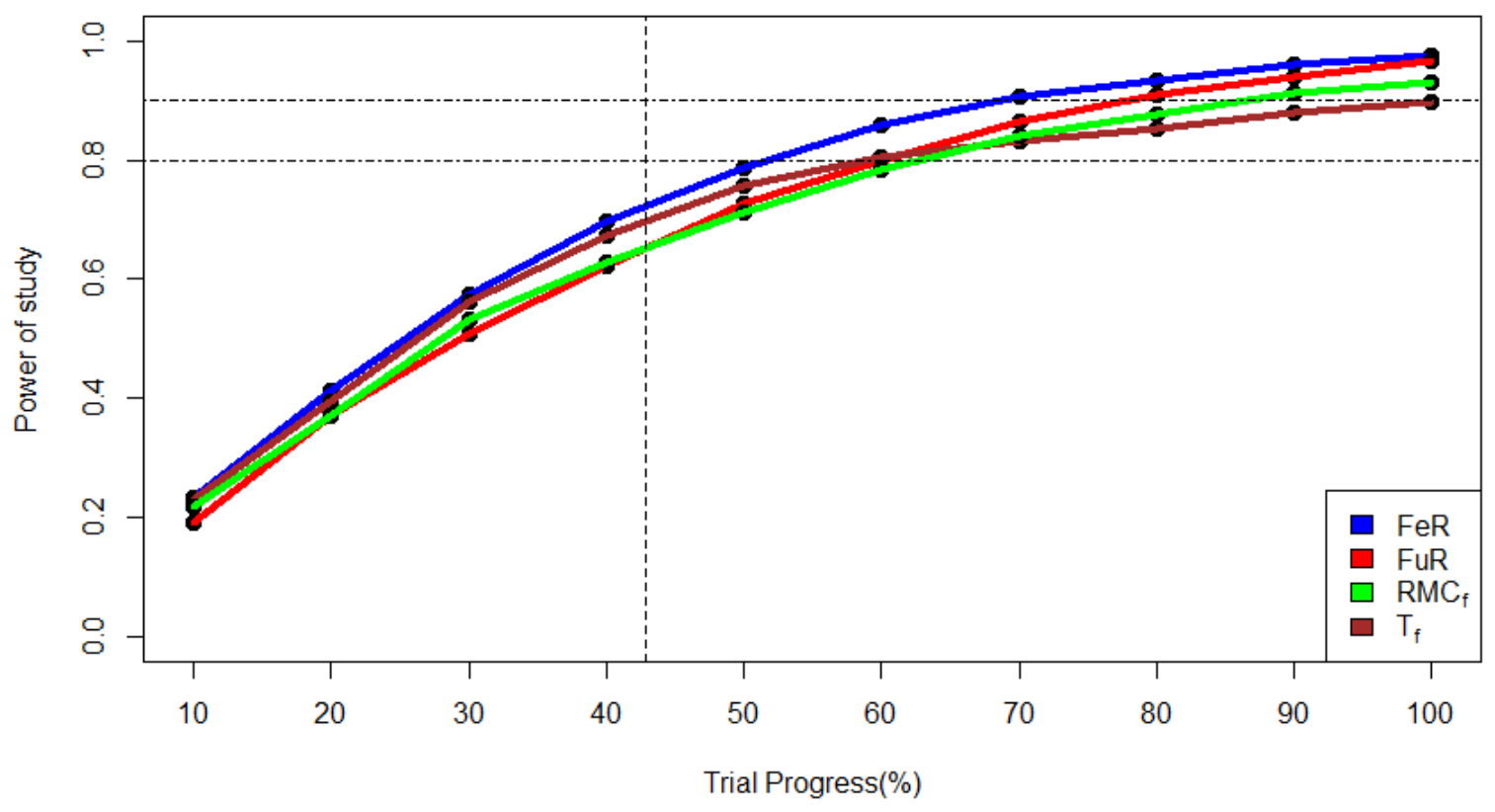



across the length of the trial. Vertical line at $43 \%$ trial progress represents the observed sample size of RECOVERY (6425). Horizontal lines represents $80 \%$ and $90 \%$ power respectively.

For subgroup-specific RAR procedures, the difference in power is minimal between randomization approaches; both $\mathrm{RMC}_{s}$ and $\mathrm{T}_{\mathrm{s}}$ produce similar power at all stages. However, there is notable differences between subgroups. Subgroup (iii) , for whom the treatment effect is largest, has the highest power and is the only subgroup where the trial reaches $90 \%$ power during the study. The other two groups only reach $80 \%$ power by the end of the study, except for Ts in the i subgroup. This is demonstrated in Figure 5.

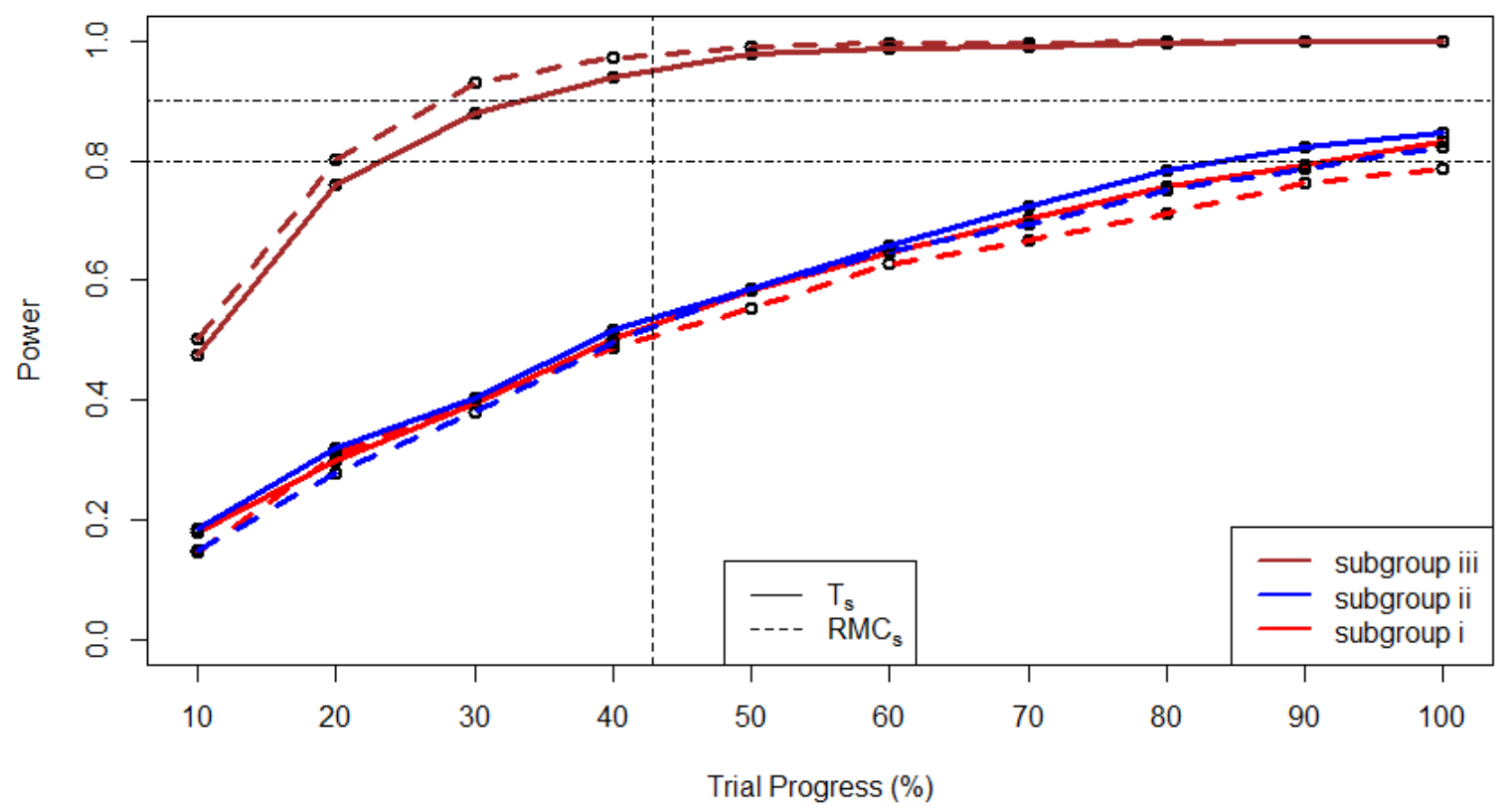

Figure 5: Expected power at each trial stage using subgroup-specific adaptive randomization. Vertical line at $43 \%$ trial progress represents the observed sample size of RECOVERY (6425).. Horizontal lines represent $80 \%$ and $90 \%$ power respectively.

\section{Bias in mortality rate estimation}

The bias associated with using cohort RAR procedures is shown for all stages of the trial in Figure 6 and in sub-group specific and cohort RAR at the point where the trial reaches the sample size 


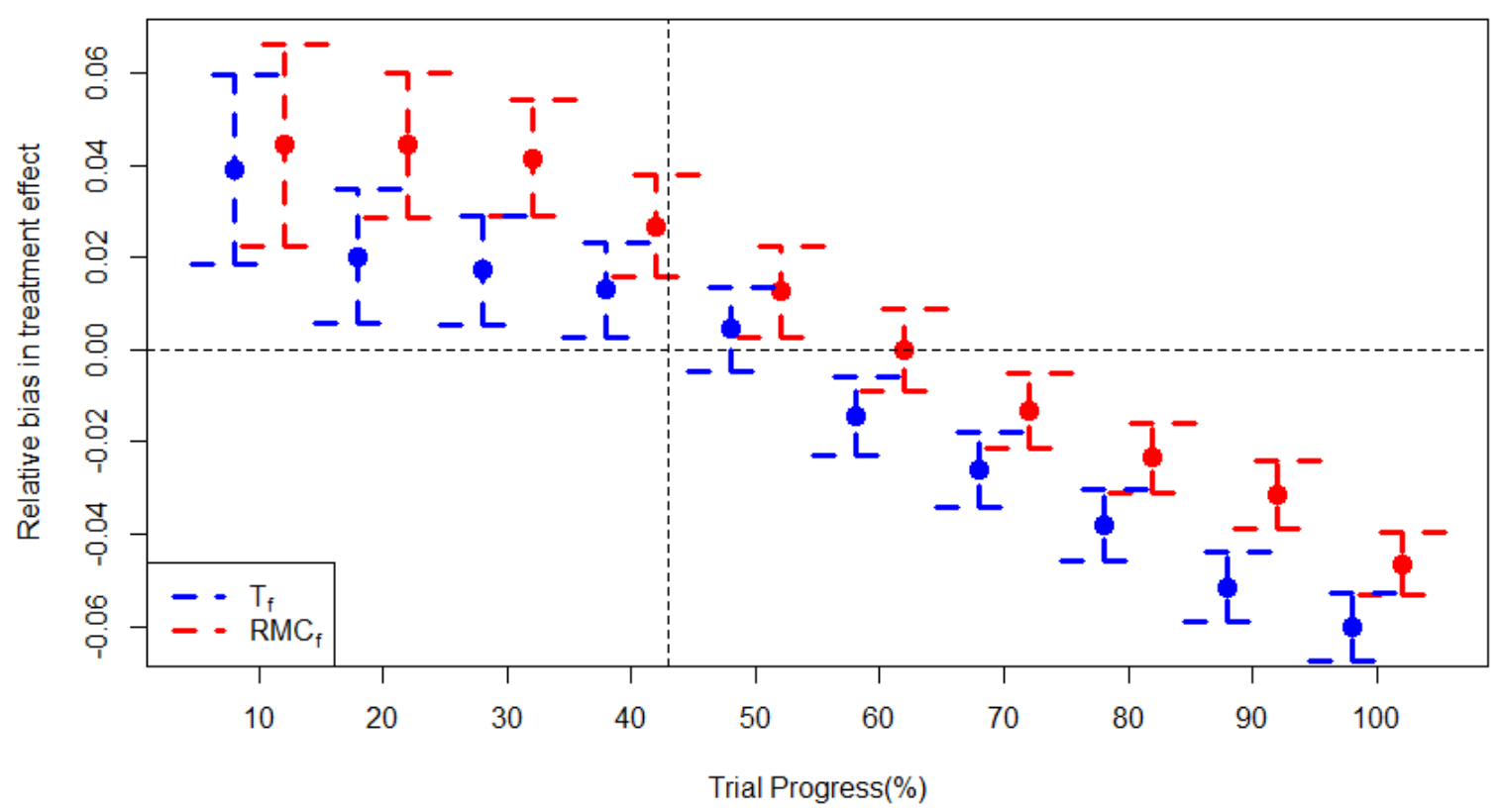




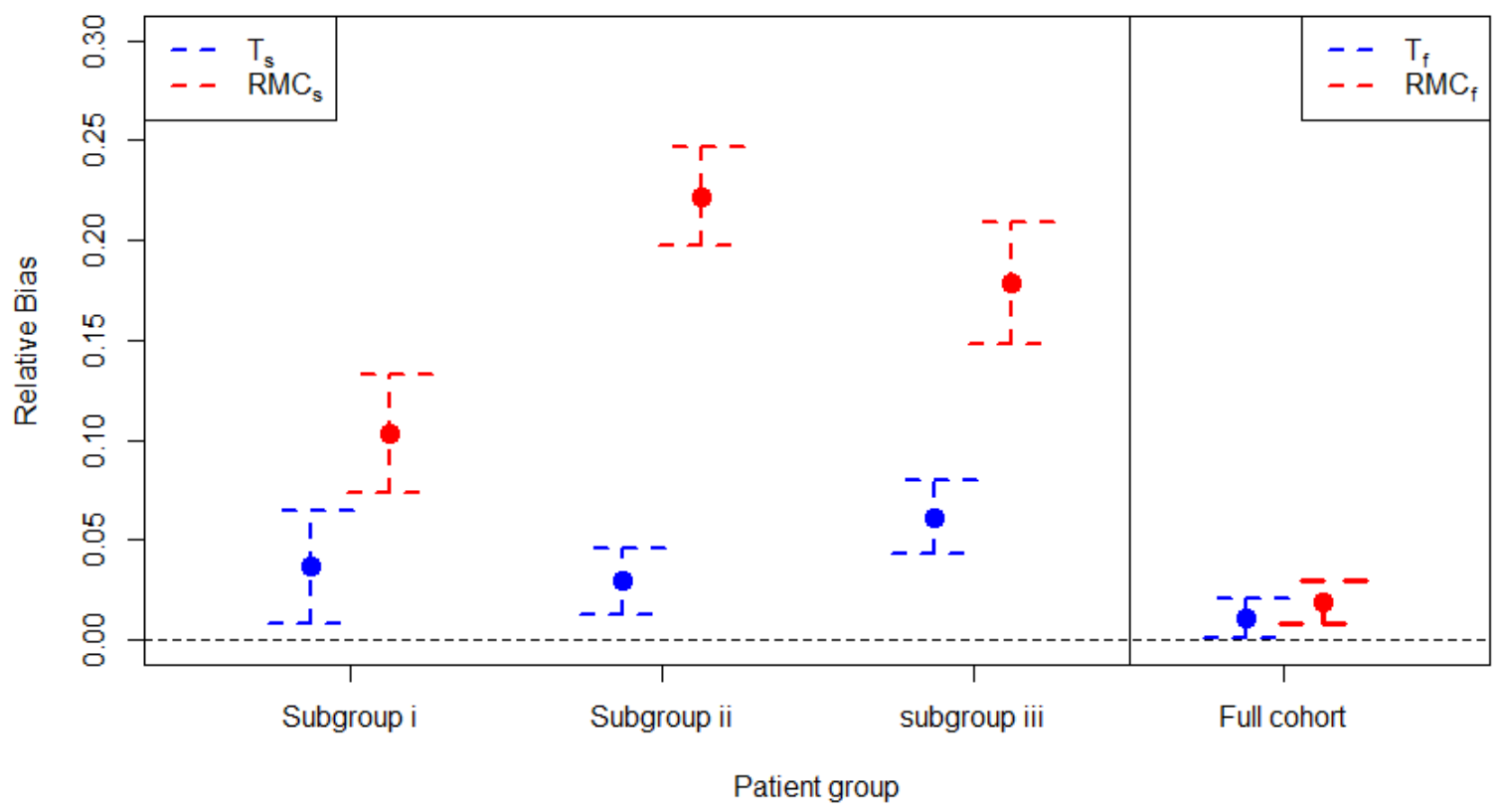

Figure 7: Bias of treatment effect estimates in the full cohort and each subgroup using the four $R A R$ schemes $\left(T_{f}, R M C_{f}, T_{s}\right.$, $R M C_{s}$ ) at the sample size observed in RECOVERY (6425).

\section{Discussion}

\section{Understanding the results}

275 It is important to consider why the different approaches led to different mortality rates. Full cohort RAR approaches led to more patients receiving dexamethasone, which was shown to be superior to standard care in most patients, and therefore led to fewer deaths overall in the trial simulation. Subgroup RAR approaches improved mortality even further because they allowed more patients in subgroup (i) to receive standard care and allowed dexamethasone allocation in subgroup (iii) to be ramped up even faster.

281 Study power is important because it indicates how many patients are likely to be required to reach 282 statistically significant results. Moreover, in the context of the pandemic, it would mean publishing 
positive results earlier which would lead to earlier use of the treatment in a real-world context. FeR showed the most study power. This is to be expected as Neyman's allocation formula indicated that the optimal allocation for study power is almost 1:1 (19). More generally, the power of all FR and cohort RAR strategies was seen to be proportional to how much their average allocation ratio skewed from 1:1. Although both FR approaches have more power than the cohort RAR approaches, it is important to mention FuR, the approach used in the RECOVERY trial, is only marginally more powerful despite leading to the most in-trial deaths. Nevertheless, the original RECOVERY trial still arguably benefitted from a larger control group because it had as many as five treatments trialled at any one time that would be compared to it.

\section{Wider implications}

Our results therefore point to a compromise between reducing in-trial patient deaths with RAR and getting statistically significant results earlier using FR. On one hand, publishing results earlier could lead to lower overall deaths outside the trial. Patients in RECOVERY accounted for only $15 \%$ of all patients with COVID-19 in hospitals (20), and the subsequent use of dexamethasone has already been estimated to have saved a million lives globally (21). On the other hand, RAR designs have been shown to improve trial recruitment, precisely because patients understand they have a higher especially true for subgroup specific RAR. While the subgroups used were declared before the start 
three groups, or whether other prespecified subgroups should be split for RAR instead. Using RAR at the subgroup level means splitting the sample, leading to smaller sample sizes in each group. This led to a low statistical power for subgroup (i) but a higher power for subgroup (iii) due to the large treatment effect. This means that the subgroup where the treatment effect is greatest benefit most from RAR.

\section{Limitations}

One important part of the model that remains unaccounted is patient drift. Patient drift occurs when the trial cohort's characteristics, and therefore their likelihood of responding to a treatment, changes throughout the course of a trial (25). When using FR, the effect of patient drift will be minimised as any changes will be independent of treatment arm allocation (e.g. if patients present later in the trial had fewer comorbidities, both the standard care and dexamethasone groups would have exhibited lower mortality rate). This is not the case when using RAR because arms that performs better will receive a larger proportion of the patients as the trial progresses. Consequently, when calculating response rates, it must be noted that the characteristics of patients could potentially be unbalanced across arms. Throughout the pandemic, data shows the type of people susceptible to catching COVID-19 has changed dramatically. This occurred in terms of age-groups, ethnicities, socio-economic class, and geography (26). Likewise, the virus itself is likely to have changed, as mutations occur, and as different claves and variants become more common (27). Resources in treating the pandemic may also change, affecting how likely patients are to survive. For example, the typical care that COVID-19 patients get varies as clinical knowledge in treating the disease improves. Additionally, if more ventilators are procured or there are fewer COVID-19 patients in a hospital, a larger proportion of patients may be placed on ventilators by doctors. This has the dual effect of making the full cohort more likely to survive as more patients can receive adequate respiratory support and making the ventilator sub-group healthier as ventilators do not have to be reserved for only the most critical patients. Therefore, had RAR been applied, it is likely 
the death rate would be skewed by the confounding effect of difference in patient, illness, and management characteristics.

Also notable is the simplicity of the simulation. RECOVERY has multiple trial arms, with arms being dropped and added dynamically. In contrast, we simulated it as a two-arm trial. Furthermore, for simplicity in the simulation, patient outcomes are generated in uniform batches. This contrasts with what happened in RECOVERY for two reasons. Firstly, the number of hospitalised patients available to recruit varied significantly throughout the trial period, and this would have affected how RAR worked. There was a sharp decrease in hospitalised patients towards the end of the recruitment period (28). This would have likely meant that there would be more information attained at the start of the trial, and therefore the proportion of patients randomised to dexamethasone would have increased faster than it did in the simulation. Secondly, there would be a delay in recording outcomes following randomization, unlike in the simulation where mortality status is recorded as soon as a patient is randomised. Although our simulation study was simplistic, we believe the results paint a broadly accurate picture of how the operating characteristics of RECOVERY would differ using FR and RAR procedures.

\section{Conclusion}

Using RAR within RECOVERY could have resulted in more patients being given the optimal treatment, and therefore fewer deaths in the trial. The benefits of RAR were even more pronounced when used within pre-specified subgroups. However, more patients would have been required to attain the same study power under RAR, leading to a longer trial period (assuming the same recruitment rate). Bias in treatment effect estimation arises in RAR trials, but only to a negligible extent. The use of RAR deserves to be considered for use in future platform trials. The trade-off in balancing the needs of patients within and beyond the trial should be acknowledged by trialists more clearly, and patient groups themselves consulted before deciding what balance to strike. 


\section{List of abbreviations}

358 RAR - Response Adaptive Randomization

$359 \quad$ FR - Fixed Randomization

360 COVID-19: coronavirus disease 2019

361 RECOVERY - Randomised Evaluation of COVID-19 Therapy

362 REMAP-CAP - Randomised, Embedded, Multi-factorial Adaptive Platform Trial for

363 Community Acquired Pneumonia

364 FeR - Fixed equal Randomization

365 FuR - Fixed unequal Randomization

$366 T_{f}-$ Tuning protocol on the full patient cohort

$367 \mathrm{RMC}_{\mathrm{f}}-$ REMAP-CAP randomization protocol on the full patient cohort

$368 T_{s}-$ Tuning protocol on each subgroup individually

$369 \mathrm{RMC}_{s}-$ REMAP-CAP randomization protocol on each subgroup individually

370 Declarations

371 Ethics approval and consent to participate

372 Not applicable.

373 Consent for publication

374 Not applicable.

375 Availability of data and materials 
Data sharing is not applicable to this article as no datasets were generated or analysed during the

377 current study.

378

379

Project name: RECOVERY RAR simulation

380

Project home page: https://github.com/ts482/RECOVERY_RAR

381

Operating system(s): Windows 10

382

Programming language: $\mathrm{R}$ statistical language

383

License: GNU GPL

384

Competing interests

385 The authors declare that they have no competing interests.

\section{Funding}

387

$\mathrm{JB}$ is funded by an Expanding Excellence in England (E3) research awarded to the University of Exeter.

TS is funded by NHS Business Services Authority through the NHS Student Bursaries scheme.

BJ is supported by the National Institute for Health Research (NIHR) Collaboration for Leadership in Applied Health Research and Care (CLAHRC) for the South West Peninsula at the Royal Devon and Exeter NHS Foundation Trust. The views expressed in this publication are those of the authors and not necessarily those of the NHS, the NIHR or the Department of Health in England. 
TS created the code simulation and wrote the first draft of the manuscript. JB contributed to the conception and design of the work, code simulation and helped edit the manuscript. BJ made substantial contributions to the conception and design of the work and helped edit the manuscript.

\section{Acknowledgements}

400

Not applicable.

401

\section{Technical appendix}

402

Technical Appenix A: Neyman rule for optimal allocation

403

404

405

406

407

408

409

410

411

412

Neyman allocation is a procedure that maximises study power for two arms given their estimated binary response rates (19). Rosenberger et al. (19) shows that optimal allocation ratio to treatment 1 for maximising power can be given by: $\frac{\sqrt{\hat{P}_{1}}}{\sqrt{\hat{P}_{1}}+\sqrt{\hat{P}_{0}}}$. In the case of the RECOVERY trial, $\hat{P}_{1}$ is the mortality rate on dexamethasone $(22.9 \%)$ and $\hat{P}_{0}$ is the observed mortality rate on standard care $(25.7 \%)$. Substituting these into the equation indicates that $49 \%$ of patients should receive dexamethasone to maximise the power of the study. Substituting the mortality rates on each treatment for the subgroups i, ii and iii (found in Table 1), the optimal allocation ratios to dexamethasone would be $53 \%, 49 \%$ and $46 \%$ respectively.

\section{Technical Appendix B: Calculating the posterior outcome probability}

To calculate the posterior probability that one treatment arm yields superior results to the other, a Bayesian beta-binomial model was assumed for each trial arm. The prior beta distribution can be given as $\pi(p \mid \alpha, \beta)=\frac{1}{B(\alpha, \beta)} p^{\alpha-1}(1-p)^{\beta-1}$, where $B(\alpha, \beta)=\int_{0}^{1} p^{\alpha-1}(1-p)^{\beta-1} d p$ and represents the Beta function. $\alpha$ and $\beta$ are set to one. The binary outcome of each patient is mortality within 28 days after randomization and can be regarded as an independent Bernoulli trial. Therefore, a sample of $\mathrm{n}$ patients follows a binomial distribution, such that $f(y \mid p)=$ 
$\left(\begin{array}{l}n \\ y\end{array}\right) p^{y}(1-p)^{n-y}$, where $\mathrm{n}$ is the total number of patients and $\mathrm{y}$ is the number of successes (or deaths within 28 days of randomization). Combining these distributions creates the posterior probability density function $\pi(\mathrm{p} \mid \mathrm{y})$, given as $\frac{1}{B(y+\alpha, n-y+\beta)} p^{y+\alpha-1}(1-p)^{n-y+\beta-1}$, which is a beta $(y+\alpha, n-y+\beta)$ distribution.

The parameters indexing the posterior beta distribution are therefore $\alpha=y+\alpha$ and $\beta=n-y+$ $\beta$. From this we can create a beta posterior distribution at each point of the interim analysis $i=$ $1, \ldots, 100$ for each trial arm $j=0$ (dexamethasone), 1 (standard care), and 100 points $k=1, \ldots, 100$. To estimate, for example, the posterior probability that the posterior mean for arm 0 is greater than the posterior mean of arm 1, we randomly generate 100 draws from each distribution and count the proportion of times that the arm 0 draw is the largest.

\section{Technical Appendix C: Bowden and Trippa rule for bias following a RAR procedure} parameter is given by:

$434 \frac{\operatorname{Cov}\left[n_{j}, \hat{P}_{j}\right]}{E\left[n_{j}\right]}=P_{j}-E\left[\hat{P}_{J}\right]=-\operatorname{Bias}\left(\hat{P}_{j}\right)$ 
1. CDC. Healthcare Workers [Internet]. Centers for Disease Control and Prevention. 2020 [cited 2021 Jun 24]. Available from: https://www.cdc.gov/coronavirus/2019-ncov/hcp/non-ussettings/overview/index.html

2. WHO Director-General's opening remarks at the media briefing on COVID-19-11 March 2020 [Internet]. [cited 2021 May 31]. Available from: https://www.who.int/directorgeneral/speeches/detail/who-director-general-s-opening-remarks-at-the-media-briefing-oncovid-19---11-march-2020

3. Coronavirus Pandemic (COVID-19) - the data - Statistics and Research [Internet]. Our World in Data. [cited 2021 Jun 1]. Available from: https://ourworldindata.org/coronavirus-data

4. Nabavi N. Long covid: How to define it and how to manage it. BMJ. 2020 Sep 7;370:m3489.

5. Whitaker M, Elliott J, Chadeau-Hyam M, Riley S, Darzi A, Cooke G, et al. Persistent symptoms following SARS-CoV-2 infection in a random community sample of 508,707 people [Internet]. 2021 Jun [cited 2021 Jun 27]. Available from: http://spiral.imperial.ac.uk/handle/10044/1/89844

6. Oncology TL. COVID-19 and cancer: 1 year on. Lancet Oncol. 2021 Apr 1;22(4):411.

7. Torales J, O'Higgins M, Castaldelli-Maia JM, Ventriglio A. The outbreak of COVID-19 coronavirus and its impact on global mental health. Int J Soc Psychiatry. 2020 Jun 1;66(4):317-20.

8. 2020 Year in Review: The impact of COVID-19 in 12 charts [Internet]. [cited 2021 Jun 27]. Available from: https://blogs.worldbank.org/voices/2020-year-review-impact-covid-19-12charts

9. University of Oxford. Randomised Evaluation of COVID-19 Therapy [Internet]. clinicaltrials.gov; 2021 Apr [cited 2021 May 27]. Report No.: NCT04381936. Available from: https://clinicaltrials.gov/ct2/show/NCT04381936

10. Dexamethasone in Hospitalized Patients with Covid-19. N Engl J Med. 2021 Feb 25;384(8):693704.

11. Abani $\mathrm{O}$, Abbas A, Abbas F, Abbas $\mathrm{M}$, Abbasi $\mathrm{S}$, Abbass $\mathrm{H}$, et al. Tocilizumab in patients admitted to hospital with COVID-19 (RECOVERY): a randomised, controlled, open-label, platform trial. The Lancet. 2021 May 1;397(10285):1637-45.

12. Group RC, Horby PW, Mafham M, Peto L, Campbell M, Pessoa-Amorim G, et al. Casirivimab and imdevimab in patients admitted to hospital with COVID-19 (RECOVERY): a randomised, controlled, open-label, platform trial. medRxiv. 2021 Jun 16;2021.06.15.21258542.

13. Recommendations | COVID-19 rapid guideline: managing COVID-19 | Guidance | NICE [Internet]. NICE; [cited $2021 \mathrm{Jul} 15$ ]. Available from: https://www.nice.org.uk/guidance/ng191/chapter/Recommendations

14. Angus DC, Alexander BM, Berry S, Buxton $\mathrm{M}$, Lewis $\mathrm{R}$, Paoloni $\mathrm{M}$, et al. Adaptive platform trials: definition, design, conduct and reporting considerations. Nat Rev Drug Discov. 2019 Oct;18(10):797-807. 
15. Pallmann P, Bedding AW, Choodari-Oskooei B, Dimairo M, Flight L, Hampson LV, et al. Adaptive designs in clinical trials: why use them, and how to run and report them. BMC Med. 2018 Feb 28;16(1):29.

16. Proschan M, Evans S. Resist the Temptation of Response-Adaptive Randomization. Clin Infect Dis. 2020 Dec 1;71(11):3002-4.

17. Interleukin-6 Receptor Antagonists in Critically III Patients with Covid-19. N Engl J Med. 2021 Apr 22;384(16):1491-502.

18. Bonten MJM. Randomized, Embedded, Multifactorial Adaptive Platform Trial for CommunityAcquired Pneumonia [Internet]. clinicaltrials.gov; 2020 Oct [cited 2021 May 26]. Report No.: NCT02735707. Available from: https://clinicaltrials.gov/ct2/show/NCT02735707

19. Rosenberger WF, Stallard N, Ivanova A, Harper CN, Ricks ML. Optimal Adaptive Designs for Binary Response Trials. Biometrics. 2001;57(3):909-13.

20. Wise J, Coombes R. Covid-19: The inside story of the RECOVERY trial. BMJ. $2020 \mathrm{Jul}$ 8;370:m2670.

21. Robinson J. Steroid has saved the lives of one million COVID-19 patients worldwide, figures show [Internet]. The Pharmaceutical Journal. [cited 2021 Aug 3]. Available from: https://pharmaceutical-journal.com/article/news/steroid-has-saved-the-lives-of-one-millioncovid-19-patients-worldwide-figures-show

22. Tehranisa JS, Meurer WJ. Can response-adaptive randomization increase participation in acute stroke trials? Stroke. $2014 \mathrm{Jul} ; 45(7): 2131-3$.

23. World Medical Association Declaration of Helsinki: Ethical Principles for Medical Research Involving Human Subjects. JAMA. 2013 Nov 27;310(20):2191.

24. Jones JH. Bad Blood. Simon and Schuster; 1993. 342 p.

25. Villar SS, Bowden J, Wason J. Response-adaptive designs for binary responses: How to offer patient benefit while being robust to time trends? Pharm Stat. 2018;17(2):182-97.

26. Venkatesan P. The changing demographics of COVID-19. Lancet Respir Med. 2020 Dec 1;8(12):e95.

27. Lauring AS, Hodcroft EB. Genetic Variants of SARS-CoV-2-What Do They Mean? JAMA. 2021 Feb 9;325(6):529.

28. UK: Daily new hospital admissions for COVID-19 [Internet]. Our World in Data. [cited 2021 Jul 27]. Available from: https://ourworldindata.org/grapher/uk-daily-covid-admissions

29. Bowden J, Trippa L. Unbiased estimation for response adaptive clinical trials. Stat Methods Med Res. 2017 Oct;26(5):2376-88. 